\title{
Optic Flow Regulation in Unsteady Environments: A Tethered MAV Achieves Terrain Following and Targeted Landing Over a Moving Platform
}

\author{
Franck Ruffier • Nicolas Franceschini
}

Received: 1 July 2013 / Accepted: 23 April 2014 / Published online: 24 July 2014

(C) The Author(s) 2014. This article is published with open access at SpringerLink.com

\begin{abstract}
The present study deals with the risky and daunting tasks of flying and landing in nonstationary environments. Using a two Degree-OfFreedom (DOF) tethered micro-air vehicle (MAV), we show the benefits of an autopilot dealing with a variable - the optic flow - which depends directly on two relative variables, the groundspeed and the groundheight. The micro-helicopter was shown to follow the ups and downs of a rotating platform that was also oscillated vertically. At no time did the MAV know in terms of ground height whether it was approaching the moving ground or whether the ground itself was rising to it dangerously. Nor did it know whether its current groundspeed was caused only by its forward thrust or whether it was partly due to the ground moving backwards or forwards. Furthermore, the MAV was shown to land safely on a platform set into motion along two directions, vertical and horizontal. This paper extends to non-stationary environments a former approach that introduced the principle of "optic flow regulation" for
\end{abstract}

Electronic supplementary material The online version of this article (doi:10.1007/s10846-014-0062-5) contains supplementary material, which is available to authorized users.

F. Ruffier $(\bowtie) \cdot$ N. Franceschini

Aix-Marseille University, CNRS, ISM UMR7287,

Biorobotics Research Group, 13288, Marseille, France

e-mail: franck.ruffier@univ-amu.fr

N. Franceschini

e-mail: nicolas.franceschini@univ-amu.fr altitude control. Whereas in the former approach no requirement was set on the robot's landing target, the target's elevation angle was used here in a second feedback loop that gradually altered the robot's pitch and therefore its airspeed, leading to smooth landing in the vicinity of the target. Whether dealing with terrain following or landing, the MAV followed appropriately the unpredictable changes in the environment although it had no explicit knowledge of groundheight and groundspeed. The MAV did not make use of any rangefinders or velocimeters and was simply equipped with a 2-gram vision-based autopilot.

Keywords Biomimetics · Optic flow · Unsteady environments · Unmanned aerial systems · Decking · Honeybees $\cdot$ MAV $\cdot$ Aerial Robot

\section{Abbreviation \\ OF Optic flow}

\section{Introduction}

Many flying animal such as insects are able to fly over non-stationary surfaces such as heavy sea, as well as to land delicately on dancing twigs, flowers blown by the wind, or floating objects in water streams. Fine skills of this kind would be most useful for autonomous aircraft that have to land on moving platforms such as shipdecks or offshore platforms. This paper extends to non-stationary environments a former approach that 
introduced the principle of optic flow regulation for an altitude control system [1,2].

The paper shows how a MAV can rely on optic flow cues to navigate above a moving platform and land on it. Strategies of two kinds are currently being used to enable an aircraft to land on a moving platform:

- Human piloted aircraft rely on qualitative information about the attitude of the landing deck during the approach and landing phases,

- Unmanned aircraft rely on both onboard and offboard data.

In the first case, an operator standing on the ship's deck informs the human pilot to decelerate or accelerate and to pitch nose up or nose down, via a set of conventional gestures. In the second case (that of an unmanned vehicle), the autopilot controls the aircraft directly, based not only on the aircraft's flight parameters (such as its position - $\mathrm{x}, \mathrm{y}, \mathrm{z}$ - as given by the GPS -Global Positioning System- receiver, and its attitude - yaw, roll and pitch - as given by the aircraft's Inertial Measurement Unit), but also on state variables relative to the moving platform itself (such as the platform's GPS position - $\mathrm{x}, \mathrm{y}, \mathrm{z}$ - and the attitude - yaw, roll and pitch - given by the platform's Inertial Measurement Unit -IMU-). In addition, some aircraft autopilots also use the aircraft's descent angle with respect to the deck as a steering parameter [3]. However, whether they are manned or unmanned, very few helicopters, even military ones, are capable of landing on the deck of a ship undergoing heavy oscillations caused by the sea swell. In recent military developments (as reported by some press releases and not published exhaustively in scientific conferences or journals so far) additional sensors based, e.g., on differential GPS, seem to have been placed on the landing platform itself. In this case, the classical autopilot therefore used the following sequence to make the aircraft land on the moving platform $[4,5]$ :

- collect current data about the position and attitude of both the platform and the aircraft using classical (active) sensors such as radar or relative GPS [3],

- determine a landing trajectory,

- servo the aircraft to this trajectory, using "optimal" [6] or "robust" [7] feedback control.

The main drawback of this approach is that it requires a full duplex radio link between the aircraft and the mobile platform. The aircraft is not at all stealthy, and all the problems generally associated with data links tend to arise, such as noise and dropouts, asynchronized data transmission, time-lags in the communications, etc. Another drawback is the large amount of computing power required to determine and consistently update the optimum landing trajectory online and transmit the results to the aircraft in terms of control commands. This would make the system hardly compatible with MAVs in the 100-milligram to 100-gram range [8-11]. Some authors have simulated an automatic landing system that generates a decking trajectory in an advanced rotorcraft environment (called FLIGHTLAB): this trajectory is the one that would be presumably taken by a human pilot on the basis of optic flow (OF) cues [12]. But here again, the trajectory was computed via the classical position and attitude data available onboard, without visually measuring the OF directly. Other authors have performed automated landing on a steady [13], and even on a moving [14] target, based on a combination of visual and inertial attitude data. Others [15] have used on-board visual processing methods based on the segmentation and localization of a colored cloth target to inform a fixed-wing MAV about the landing spot targeted in angular terms in the local frame of reference. Others have used real video data and intensive optic flow computations to reconstruct the scene offline while a UAV was approaching a landing target [16]. The optic flow-based autopilot presented here was originally inspired by findings made on flying insects, which led to the development of the "Optic flow regulator" concept $[1,2]$. More specifically, the work presented here was triggered by our recent observations of honeybees that we made fly over a moving floor [17], and by the impressive feats they achieve when landing on a moving target [18].

Several OF-based guidance systems have been developed over the years to use onboard terrestrial and aerial robots. During the last decade, some fixedand rotary-wing aerial robots have been equipped with similar means of detecting and processing the OF to those providing insects such as flies and bees with the vital cues they need to take off, follow a terrain and land (blimp: [19], fixed-wings: [8, 20-22], rotary-wings: [1, 2, 9, 23-25]), to hover (rotary-wings: [10, 26, 27]), and to track a moving target (rotary-wings: [27-29]). In aerial robots, OF 
processing methods were used in several ways as ameans of:

- estimating the state variables of the system with respect to the inertial reference frame along with other more classical sensors such as Inertial Measurement Units (IMUs), sonars, GPS, airspeed sensors and/or accelerometers [27, 30, 31] :

- extracting relative-state information for navigation purposes using the Wide Field Integration method [32], or

- using the OF directly in a feedback control loop without any need for information about the velocity, acceleration, altitude or even about the characteristics of the terrain. Some OF based aerial robots are able to perform tasks such as take-off, terrain-following and safe landing [1, 2, 33] as well as avoiding frontal obstacles [34].

The latter OF-based approaches to achieve risky tasks differ considerably from the conventional approach where all the states of the aerial robot are either measured or estimated in the inertial reference frame $[35,36]$.

Very few attempts have been made so far to apply OF techniques to flight guidance over nonstationary environments. Recently, a free-flying quadrotor succeeded in performing vertical landing on a vertically oscillating platform by relying on vertical optic flow expansion cues [37, 38]. Although the rotorcraft used by these authors was much heavier, OF computation was not performed onboard. Additionally, a miniature quadcopter was shown to land accurately on a moving carrier vehicle equipped with a pattern of infra-red lights [39].

In previous papers $[1,2]$, the OCTAVE robot was regulating its ventral optic flow by modifying its rotor-speed and hence its height over a stationary environment while a human operator set its pitch (at a constant or ramp-wise level). The rotorcraft had no requirements on where it was to land. In the present paper, the flying robot regulates its ventral optic flow by bobbing up and down over the vertically oscillating platform. In the last section, the robot is equipped with an additional feedback loop that makes it automatically decrease its pitch, and hence its forward speed, as a consequence of approaching the target. We show here that the aerial robot is able to land near a virtual target on the sole basis of the ventral OF value and the target's elevation angle $\alpha$ (see Fig. $8 \mathrm{~b}$ and
Eq. 11), without the need for any metric measurements such as groundspeed or groundheight. Flying and landing in an unsteady environment is such a challenging task that we found essential to use a testrig limiting the flight trajectory to the longitudinal plane in this early stage where the stake was to experiment new algorithms and new principles in a safe manner.

In Section 2, the ventral "optic flow regulator" principle [1] is introduced with specific reference to an unsteady platform causing two types of disturbances, in the horizontal and vertical directions. Section 3 describes the experimental set-up in which a miniature tethered rotorcraft measuring its optic flow on-board is made to fly over a circular platform set in motion in both the forward and upward directions. Section 3 also discusses the alteration in flight dynamics caused by the flight mill and the limitation of the system to low forward velocities. Section 4 shows how the optic flow regulator driving the lift enables the tethered rotorcraft to safely follow a non-stationary platform that is set in motion both horizontally and vertically. Lastly, in Section 5, a second feedback loop driving the forward speed on the basis of the target's elevation angle brings the rotorcraft to land near the virtual target on the horizontally and vertically moving platform.

\subsection{Biorobotics Contribution}

This study belongs to the field of biorobotics, where robots are often used as models for explaining animal behaviour [40]. We show that honeybees' behaviour can be modelled on the basis of optic flow measurements when they are flying and landing over a platform moving up and down. The responses of this model to the uncertainties inherent to real environments, real actuators and real sensors were tested by implementing it onboard a real physical rotorcraft. In the present study, the navigation performances of the OCTAVE robot were tested over an unsteady ground set into both horizontal and vertical motion. The horizontal, forwards or backwards movement of the ground perturbed the robot's groundspeed in the same way as a head wind or tail wind, respectively. In a nutshell, the control scheme underlying the rotorcraft's behaviour accounts for flying [17, 41] and landing [18] abilities observed in bees in wind or nonstationary conditions, whereas previous bioinspired 
robots do not account for all these bees' performances. While providing valuable ideas for MAVs' navigation in unsteady environments, our results generate new hypotheses for designing further biological experiments, the results of which will help in turn to improve the model and then future robots.

\section{Optic Flow-based Visual Guidance Above an Unsteady Platform}

Here we present the principles governing the movement of a micro-rotorcraft flying in translation over an oscillating surface, focusing on the tethered rotorcraft's movements in the longitudinal plane. After simplification of the general equation from [42], since the tethered rotorcraft is equipped with an artificial eye that can be oriented downward towards the moving platform, the Optic Flow (OF) perceived by this eye depends on the following equation:

$\omega_{\varphi}=\frac{V_{M A V / \text { deck }}}{D} \sin \varphi$

where $V_{M A V / \text { deck }}$ is the speed of the rotorcraft with respect to the platform below, $D$ is the distance from the robot's eye to the downward platform in the lineof-sight direction, and $\varphi$ is the angle between the line-of-sight direction and the speed vector. When the rotorcraft's eye is oriented vertically downwards, $V_{M A V / \text { deck }} \cdot \sin \varphi$ becomes $V_{x_{M A V / d e c k}}$ and $D$ becomes $h_{M A V / d e c k}$, the robot's height above the platform. This defines the ventral optic flow $\omega_{v t r}$ which depends simply on the ratio between the horizontal speed with respect to the surface below $V_{x_{M A V / d e c k}}$ and the robot's height above the surface $h_{M A V / d e c k}$ (or eye-height), as follows:

$\omega_{v t r l}=\frac{V_{x_{M A V / d e c k}}}{h_{M A V / \text { deck }}}$

As described in [1], the eye can always be oriented directly downwards by means of an accessory microservo system, regardless of the rotorcraft's forward pitch, and the eye can measure the 1-D optic flow by means of a neuromorphic 2-pixel local motion sensor (also called EMD). This fly-inspired 2-pixel local motion sensor assesses the optic flow on the basis of the inverse of the delay between the detection of the same contrasting feature by two adjacent photoreceptors [24, 43]. This 1D optic flow estimation method is largely invariant to contrast and spatial frequency [1].
We previously developed the optic flow regulator concept that enables a robotic micro-helicopter (Fig. 2a) to perform challenging tasks such as take-off, terrain following, and landing in steady environments $[1,2,44,45]$. The basic components of this autopilot are an Optic Flow (OF) sensor that measures the ventral $\mathrm{OF}, \omega_{\text {meas }}$, and a feedback loop called the ventral OF regulator (Fig. 3), which strives to maintain a constant $\omega_{\text {meas }}$ value equal to a set-point $\omega_{\text {set }}$. A comparator produces an $\mathrm{OF}$ error signal $e_{\omega}$, which drives a lead controller modifying the lift, and thus the groundheight, so as to minimize the OF error signal. The operator only has to set the pitch angle $\theta$ (Fig. 3), and the OF regulator does the rest: in other words, it keeps the $\frac{V_{x_{M A V / d e c k}}}{h_{M A V / d e c k}}$ ratio constant in the steady state (see Eq. 2). In the next, we will see that this same OF regulation scheme is able to make the robot fly over an oscillating and rotating platform without any needs to measure groundheight and groundspeed, as shown in the video (Online Resource 1).

\section{Experimental Set-Up}

\subsection{Aerial Robot}

Figure 2A shows the aerial robot equipped with its ventral optic flow (OF) sensor and its visuo-motor control electronics: this tethered micro-helicopter was developed for testing OF-based autopilots. The propulsion unit of the original robot [24] was improved by introducing a miniature brushless motor (Megax Brushless Mb 12-3017) and a 1:15 reducer. A protective shield made of thin carbon rods was placed around the robot, which brought the eye $25 \mathrm{~cm}$ above the deck platform when the rotorcraft had landed. As previously described in detail [1], the neuromimetic visual motion sensor kept looking vertically downwards, regardless of the rotorcraft's pitch.

\subsection{Test-Rig}

The rotorcraft was tethered to the end of a light, counterbalanced pantographic flight mill (Fig. 2b), which was free to rotate frictionlessly in azimuth and elevation about a central pole. The heave and surge dynamics were controlled by the rotorcraft's vertical 


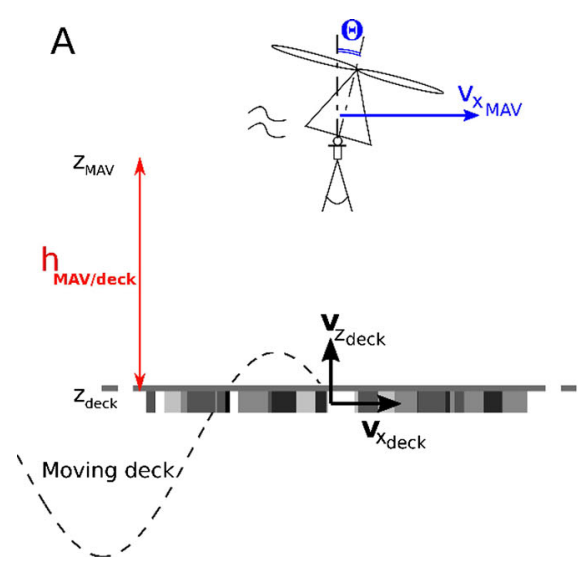

Fig. 1 Incoming ventral Optic Flow (OF), $\omega_{\text {vtrl }}$ and ventral Optic Flow measured $\omega_{\text {meas }}$ by an aerial robot flying in translation over a platform, or deck, that is itself moving both horizontally and vertically. a The ventral OF perceived by an aerial robot flying at the horizontal groundspeed $V_{x_{M A V / d e c k}}$ and the height $h_{M A V / d e c k}$ with respect to the surface below is the angular speed $\omega_{v t r l}$ at which a point in the surface texture directly below seems to move in the opposite direction.

lift and horizontal propulsive forces, respectively. The rotorcraft's lift produced by the rotor elevates its own mass while a counterweight compensates for the mass of the flight mill. An external servo-motor pitching the aerial robot forwards or backwards can be remotely controlled by either the operator (see Section 4) or

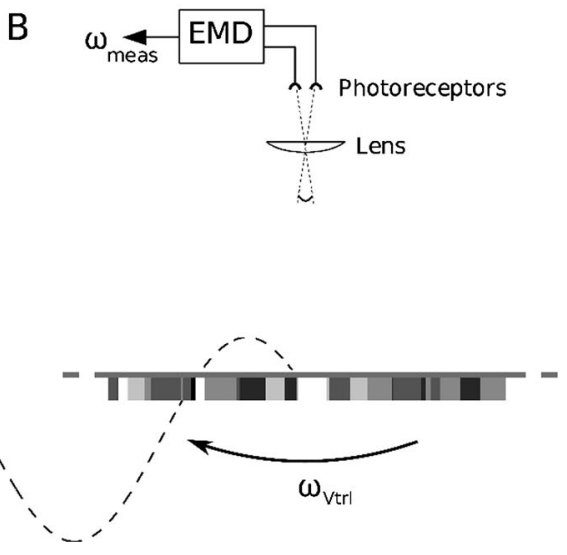

By definition, the ventral optic flow $\omega_{v t r l}$ depends on the current groundspeed-to-groundheight ratio where the groundspeed is actually the rotorcraft horizontal speed relative to the moving platform. b The minimalistic "OF sensor" used onboard the aerial robot comprises a microlens and two photoreceptors driving a fly-inspired elementary motion detector (EMD). The output $\omega_{\text {meas }}$ from the OF sensor serves directly as a feedback signal in the control scheme shown in the Fig. 3

an additional autopilot (see Section 5), depending on the experiment. Since the robot's pitch angle $\theta$ departs only slightly from the vertical (never exceeding 10 degrees), the vertical lift and the horizontal thrust can be said to be driven by the rotor's speed and the pitch angle, respectively.

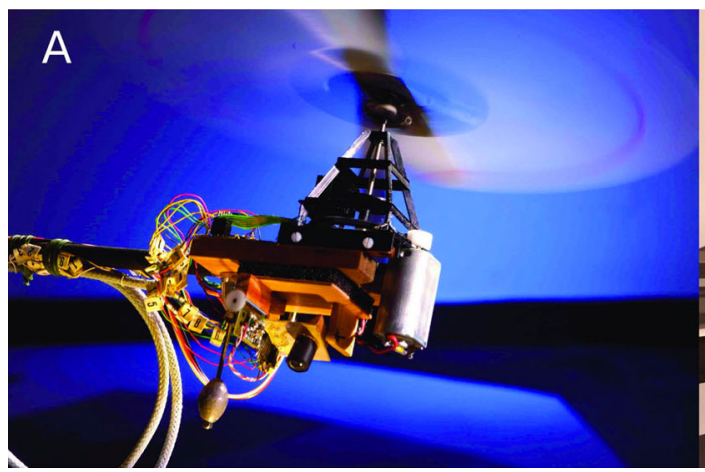

Fig. 2 Tethered MAV and experimental rig showing the rotorcraft flying over a moving 2-Degree-Of-Freedom (DOF) platform that can be set in motion both horizontally and vertically. a The 100-g sighted MAV was developed for testing our OFbased autopilot. Whenever the robot's pitch was modified, the onboard micro-servo automatically counter-rotated the eye so as to keep the line-of-sight oriented vertically downwards, as shown in Fig. 1A 1. b The 100-g rotorcraft was tethered to the

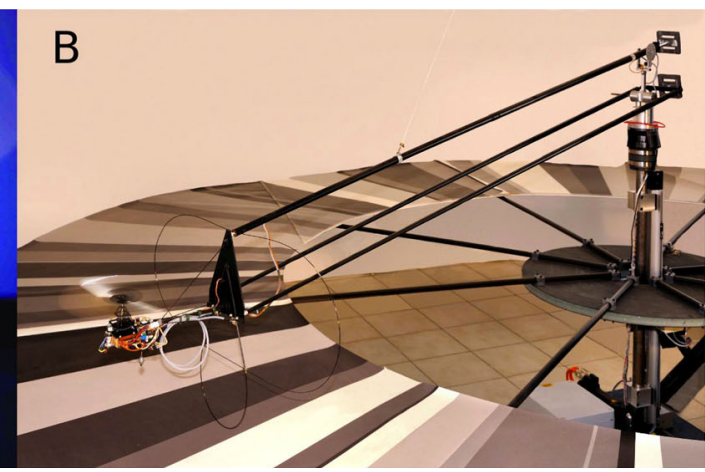

tip of a light pantographic arm (radius $1.9 \mathrm{~m}$ ) that was free to rotate frictionlessly around a central pole. The aerial robot generates its own lift and is free to fly around with an unhindered, unlimited course (average track length per lap $=12 \mathrm{~m}$ ) above a flat platform covered with a richly contrasting pattern. This flat textured platform can be actuated independently to make it rotate, and rise or fall (photograph in A: courtesy of H. Raguet) 


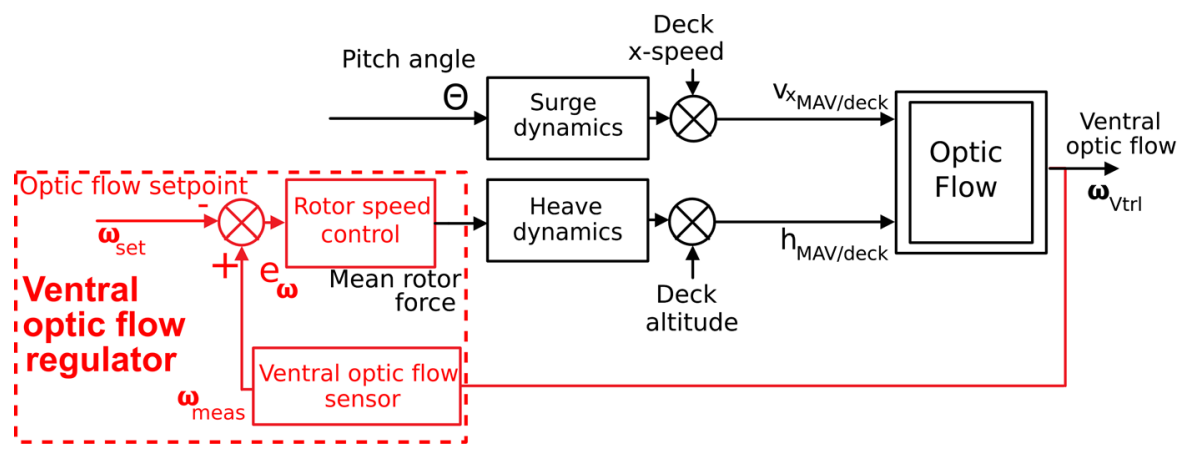

Fig. 3 The ventral "Optic-flow regulator" (red scheme) makes the aerial robot adjust its altitude, depending on the movements of a platform that can move both back-and-forth and up-anddown. The OF regulator strives to adjust the lift so as to keep the ventral OF $\omega_{\text {vtrl }}$ constant at all times. This block diagram

3.3 Influence of the Flight Mill on the Dynamics of the Aerial Robot

\subsubsection{Simplified Heave Dynamics of a Free-Flying Rotorcraft}

In the case we only take into account the lift $\left(F_{\text {Rotor Lift }}\right)$, the weight, and the viscous friction along the $\mathrm{z}$ axis, the form of the heave transfer function of a free-flying robot is the following:

$\frac{\Delta z}{\Delta F_{\text {RotorLift }}}(s)=\frac{k}{s(1+\tau s)}$

Therefore, in a first approximation, the dynamics of a free-flying rotorcraft involves a pure integrator.

\subsubsection{The Influence of the Inertial Forces on the Tethered Aerial Robot}

There is negligible influence of the gyroscopic torque because the MAV's rotor is nearly coaxial to the central pole. In contrast with the case of a free-flying robot, the circling of the MAV produces centrifugal forces linked to the mass of the robot, the pantographic arm and the counterweight.

We can write the sum of the linear momentum produced by the centrifugal forces that depends on the arm inclination $\beta$ with respect to the vertical, as follows:

$$
\begin{aligned}
& p_{\Sigma F_{\text {Centrifugal }}}=\left(\left(r \cdot F_{C t r f g l_{\text {Robot }}}\right)+\left(r / 3 \cdot F_{C t r f g l_{A r m}}\right)\right. \\
& \left.+\left(r / 12 \cdot F_{C t r f g l_{C n t r W g h t}}\right)\right) \cdot \cos \beta
\end{aligned}
$$

gives the causal and dynamic relationships between the sensory and motor variables and motor variables. In contrast with former studies [1, 2], here, deck-speed and deckaltitude are both time-dependent perturbations

where:

- $r=1.8 m$ is the radius of the pantographic arm,

- $r / 3=0.6 m$ is the distance between the arm's center of mass and the central pole,

- $\quad r / 12=0.15 m$ is the distance between the counterweight's center of mass and the central pole,

- $\quad F_{\text {Ctrfgl }}$ Robot $=m_{\text {Robot }} \cdot r \cdot \sin \beta \cdot \Omega_{z_{\text {arm }}}^{2}$,

- $F_{C t r f g l_{\text {arm }}}=m_{\text {arm }} \cdot r / 3 \cdot \sin \beta \cdot \Omega_{z_{\text {arm }}^{2}}^{2}$,

- $F_{C t r f g l_{\text {CntrWht }}}=m_{C n t r W g h t} \cdot r / 12 \cdot \sin \beta \cdot \Omega_{z_{\text {arm }}}^{2}$.

When the arm is inclined by $\beta=45^{\circ}$ with respect to the vertical, the linear momentum produced by the centrifugal forces $p_{\Sigma F_{\text {Centrifugal }}}$ is maximum as follows:

$p_{\Sigma F_{\text {Centrifugal }}} \leq 0.16 N . m$

with $v_{x_{M A V}}=1.5 \mathrm{~m} / \mathrm{s}, m_{\text {Robot }}=0.1 \mathrm{~kg}$ and $m_{\text {CntrWght }}=2 \mathrm{~kg}$

The linear momentum produced by the robot mass is the following:

$p_{W_{\text {Robot }}}=m_{\text {Robot }} \cdot g \cdot \sin \beta \cdot r=1.26 N . m$

If we compare the momentum produced by the robot mass and the momentum produced by the centrifugal forces, we can say that the centrifugal forces are negligible as long as the tethered robot is flying below 1.5 meters per second.

$\frac{p_{\Sigma F_{\text {Centrifugal }}}}{p_{W_{\text {Robot }}}} \leq 0.13$ 


\subsubsection{Heave Dynamics of a Tethered Aerial Robot}

We have conducted a mechanical analysis of the equilibrium points of a counterbalanced pantographic arm equipped with tethered aerial robot and we have found that an elastic restoring and stabilizing torque makes the robot always come back to its sole strictly stable equilibrium position when it moves aside from this equilibrium point (exactly like in the Roman steelyard problem) (see Section 5.4 of [46] for further details). By applying the second Newton's laws of motion along the inclination axis on the counterbalanced pantographic arm equipped with the rotorcraft, we have shown that the heave dynamics can be described by a second order transfer function as follows (see Section 5.4 of [46] for further details):

$$
\frac{\Delta z}{\Delta F_{\text {RotorLift }}}(s)=\frac{K_{z} \cdot \omega_{z}^{2}}{s^{2}+2 \zeta_{z} \omega_{z} s+\omega_{z}^{2}}
$$

We notice here that, in contrast with the situation in the tetherless MAV (Eq. 3), the heave transfer function of the tethered aerial robot does not include a pure integrator. By collecting robot's altitude data in response to a step of rotor's speed, we identified the following parameters: $K_{z}=0.00512$ meters/rotor rpm, $\zeta_{z}=$ 0.223 and $\omega_{z}=0.951 \mathrm{rad} / \mathrm{second}$.

Given that the natural angular frequency $\omega_{z}$ is slow and the integrator is absent, we conclude that if the presence of the tether adversely alters the heave

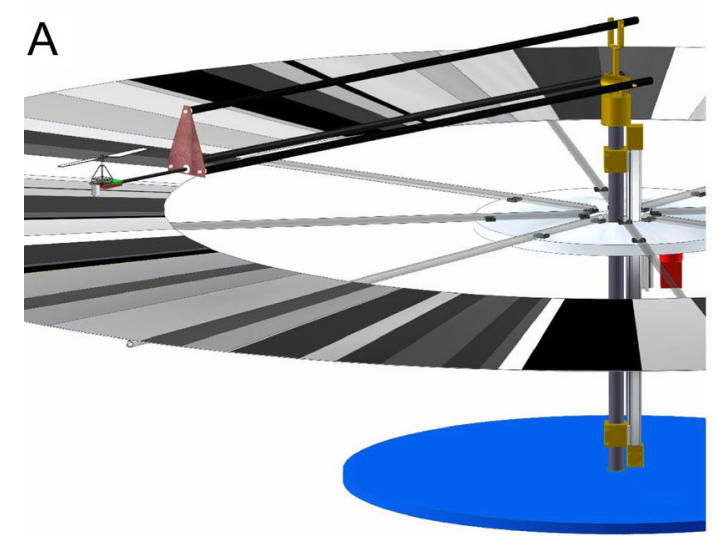

Fig. 4 Test-rig composed of a pantographic flight mill supporting the $100 \mathrm{~g}$ rotorcraft, which flies over a circular platform with a $4.5 \mathrm{~m}$ outer diameter. The platform is covered with richly textured paper: a one-dimensional computer-printed pattern with a random spatial wavelength $\lambda(0.57 \mathrm{deg} \leq \lambda \leq 16.7 \mathrm{deg}$ at $\mathrm{h}=1 \mathrm{~m})$ and a random contrast $m(0.04 \leq m \leq 0.3)$. The dynamics, making the robot less agile, it affords invaluable advantages in terms of accurate recording, reproducibility and safety of the flight trajectories. The robot's limited agility does not ultimately impair the demonstration of terrain following and landing on an unsteady platform.

\subsection{The 2-Degree-Of-Freedom Moving Platform} (Fig. 4)

A two-degree of freedom (2-DOF) moving platform was constructed, using printing paper glued onto eight sectors of $6 \mathrm{~mm}$-thick light foam (Depron) forming a disc with an outer diameter of $4.5 \mathrm{~m}$. This lightweight contrasting disc was supported by eight 2-meter long carbon fiber spokes fixed to a large central hub (diameter $0.8 \mathrm{~m}$ ). Here we describe how the vertical and horizontal degrees of freedom were controlled independently:

- The operator could make the platform oscillate vertically and tune the frequency and the amplitude of the vertical movements independently up to a maximum rise or descent speed of $300 \mathrm{~mm} / \mathrm{s}$ and a maximum amplitude of $660 \mathrm{~mm}$. The vertical movements were triggered by a linear drive unit Wiesel Speedline WH4O composed of a ball bearing guidance system, a sliding carriage and a toothed belt drive. The linear drive unit was

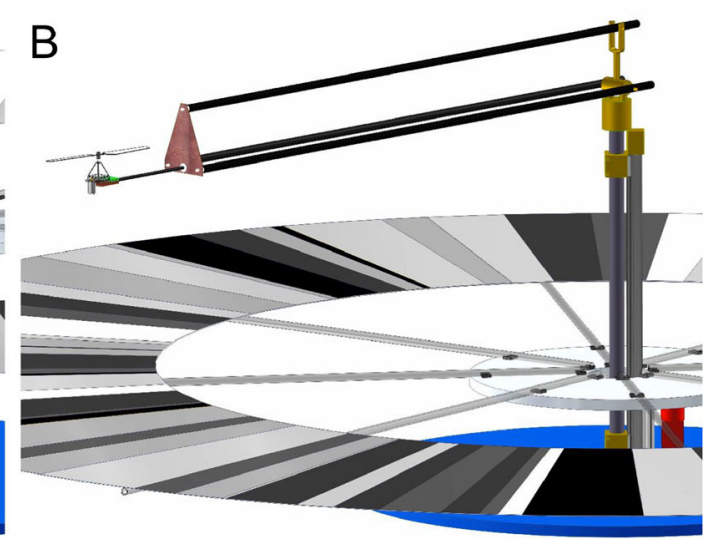

OF-based autopilot can be perturbed by moving the textured platform both forwards-and-backwards and up-and-down. a The textured landing platform in the highest position, $25 \mathrm{~cm}$ above the resting state. b The landing platform in the lowest position, $25 \mathrm{~cm}$ below the resting state 
attached vertically to the central pole and coupled to a Kollmorgen brushless motor (diameter: $50 \mathrm{~mm}$; length: $213 \mathrm{~mm}$ ) equipped with a planetary gear reducer, a multi-turn absolute encoder and a safety braking system, and driven by an industrial Servostar controller.

- The platform could also be set in horizontal motion about the central pole and its speed could be closely controlled in both the forward and backward directions. A $62 \mathrm{~mm}$-diameter Crouzet brushless motor mounted vertically on the vertical sliding carriage caused the platform to rotate about the central pole via a gearing system and a slim ball-bearing (Kaydon, inner diameter: $150 \mathrm{~mm}$, outer diameter: $160 \mathrm{~mm}$ ) concentric with the central pole, supporting the hub and its radial spokes. A torque limiter was placed between the Crouzet motor and the gearing system for safety purposes.

\section{Cruise Flight Over a Vertically and Horizontally Moving Platform}

\subsection{Flight Parameters}

The OF signal measured $\omega_{\text {meas }}$ was the output signal (in Volts) from the airborne OF sensor [1]. The rotorcraft's flight trajectory was monitored in real time from the output signals of two ground-truth sensors, both of which were mounted on the rotational axes of the pantographic arm:

- a servo-grade miniature potentiometer monitoring the rotorcraft's elevation, and

- an optical encoder monitoring the rotorcraft azimuthal position.

The platform motion was also monitored in real time from the output signals of two further ground-truth sensors:

- a micro-compass mounted on the moving platform and transmitting the azimuthal position of the platform to the base station via a HF radio link (frequency $433 \mathrm{MHz}$ ), and

- a multi-turn absolute encoder (coupled to the Kollmorgen brushless motor of the vertical linear drive) monitoring the platform's altitude $z_{\text {Deck }}$.
The measurements were converted into MAV altitude $z_{M A V}$, MAV horizontal distance travelled $x_{M A V}$, MAV horizontal airspeed $V_{x_{M A V}}$ and platform horizontal speed $V_{x_{\text {deck }}}$. The robot's trajectory was also monitored in terms of relative flight parameters such as the MAV groundheight relative to the platform $h_{M A V / \text { deck }}$ and the MAV's groundspeed relative to the platform $V_{x_{M A V / d e c k}}$, which were computed as follows:

$h_{M A V / \text { deck }}=z_{M A V}-z_{\text {deck }}$

$V_{x_{M A V / d e c k}}=V_{x_{M A V}}-V_{x_{\text {deck }}}$

Although the experiments presented in this section were all preceded by simulated trials performed under similar conditions, the data show only the actual flight paths and flight parameters monitored on the real physical helicopter. The experiments presented in this first series show the helicopter's flight behavior when its optic flow $(\mathrm{OF})$ regulator is perturbed by two types of movement of the platform: the platform forward speed and the platform altitude. These two perturbations affect the optic flow regulator at two different sites (see Fig. 3). Figures 5, 6 and 7 show how the rotorcraft equipped with the optical flow regulator described in Fig. 3 adjusted its altitude in response to a non-stationary environment: the platform was made to move up and down sinusoidally along its vertical axis at a frequency of $0.1 \mathrm{~Hz}$, with a peak-to-peak amplitude $\left(z_{\text {deck }}^{\text {peak-to-peak }}\right)$ of 0.3-meter or 0.5-meter, depending on the experiment. In this first series of experiments (Figs. 5, 6 and 7), the rotorcraft's pitch angle $\theta$ was kept constant, causing the MAV to always fly at a constant horizontal airspeed $V_{x_{M A V}}$. In Fig. 5, the platform was set in motion only in the vertical direction, and the OF regulator can be seen to have neatly rejected this ventral optic flow perturbation, keeping the original OF nearly constant (Fig. 5d, e) by successively increasing and decreasing the rotorcraft's altitude $z_{M A V}$ in line with the sine wave movements of the platform (Fig. 5a). In Fig. 6, the platform was not only set in motion vertically but also made to move forward horizontally $V_{x_{\text {deck }}}>0 \mathrm{~m} / \mathrm{s}$ ), thus causing the MAV's groundspeed relative to the platform $V_{x_{M A V / d e c k}}$ to decrease substantially. The aircraft therefore again rose and descended with the wave, at a groundheight above the platform $h_{M A V / d e c k}$ that was proportional to the relative groundspeed $V_{x_{M A V / d e c k}}$, i.e., at a lower groundheight than in the previous experiment. This response automatically restored the 

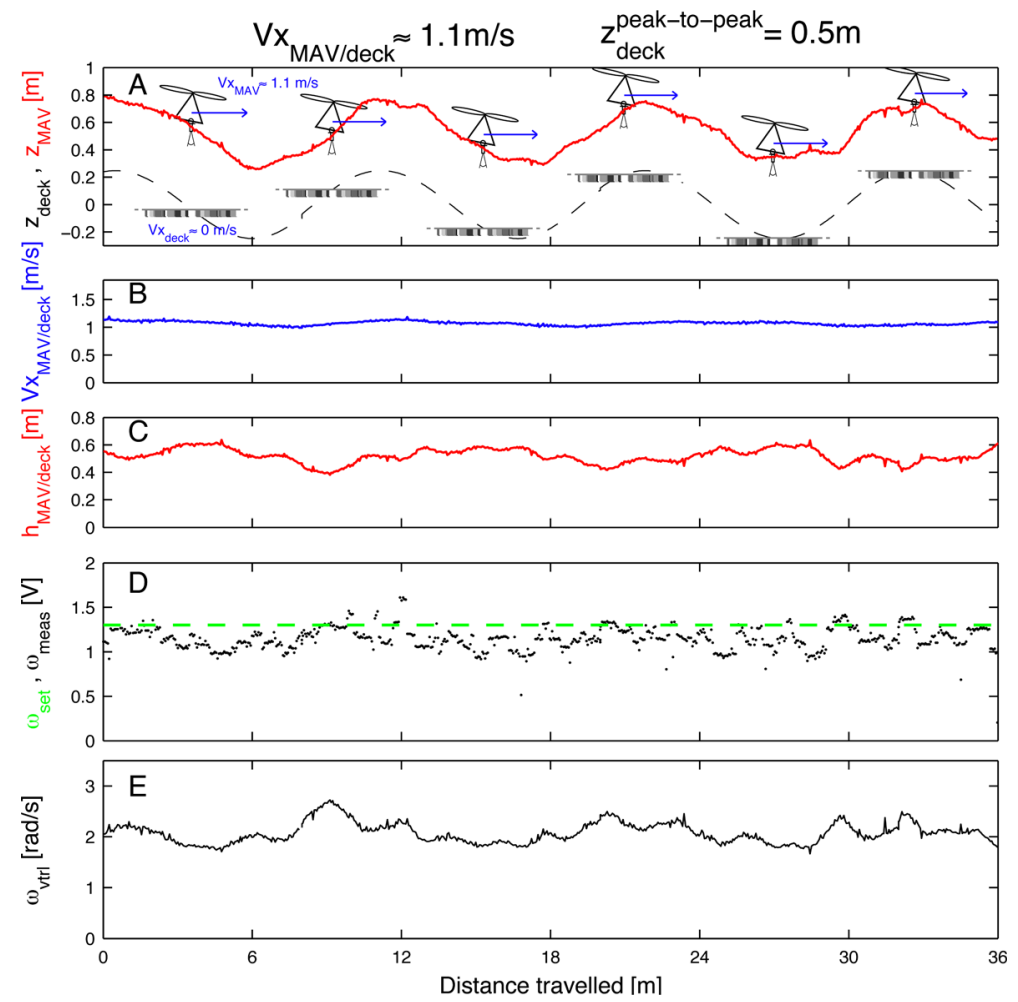

Fig. 5 Flight parameters of the robotic micro-helicopter flying at $1.1 \mathrm{~m} / \mathrm{s}$ over a non-rotating but vertically oscillating platform. a Vertical trajectory $z_{M A V}$ of the MAV equipped with a ventral OF regulator (Fig. 3). The $0.5 \mathrm{~m}$-peak-to-peak amplitude vertical oscillations of the platform $\left(z_{\text {deck }}^{\text {peak-to-peak }}\right)$ made the MAV automatically increase and decrease its altitude with the wave, so as to maintain the ventral OF constant. The MAV hugged the platform by following its large amplitude oscillations faithfully. b Since the robot's pitch was constant and the platform did not move horizontally $\left(V_{x_{\text {deck }}}=0 \mathrm{~m} / \mathrm{s}\right)$, the groundspeed $V_{x_{M A V / d e c k}}$ was always $1.1 \mathrm{~m} / \mathrm{s}$ throughout the journey. c To maintain the OF constant, the autopilot adjusted the lift to make the relative

ventral $\mathrm{OF}$, in much the same way as honeybees restore their ventral OF when they descend over a forward moving pattern [17]. In Fig. 7, the platform was moved backwards $\left(V_{x_{\text {deck }}}<0 \mathrm{~m} / \mathrm{s}\right)$, causing the horizontal groundspeed relative to the platform $V_{x_{M A V / d e c k}}$ to increase. The MAV therefore again rose and fell with the wave, but this time at a greater groundheight relative to the platform $h_{M A V / \text { deck }}$. The new groundheight was again proportional to the relative groundspeed $V_{x_{M A V / d e c k}}$, and it again restored the ventral OF. In all the cases presented here, the rotorcraft can be seen to have flown in line with the wave and to have followed the moving platform by automatically groundheight $h_{M A V / \text { deck }}$ always proportional to the groundspeed. Because the latter was constant here, the groundheight relative to the platform was observed to be constant as well, at about $0.5 m$ throughout the journey, despite the large amplitude of the platform's vertical oscillation. $\mathbf{d}$ The $\mathrm{OF} \omega_{\text {meas }}$ measured by the OF sensor was monitored throughout the journey and showed only minor deviations from the OF set-point $\omega_{\text {set }}$, which was 1.3 Volts here (corresponding to a visual angular speed of $\approx 125 \mathrm{deg} / \mathrm{s}$ ). e The actual $\mathrm{OF}$, calculated as the ratio $V_{x_{M A V / d e c k}}: h_{M A V / \text { deck }}$ also remained constant throughout the flight path

and safely adjusting its clearance from the platform, as dictated by its OF regulator.

\section{Landing on a Moving Platform: Strategy and Results}

The target elevation sensor is simulated by computing an elevation angle $\alpha$ (Fig. 8b) using several variables. The horizontal distance to be travelled up to the target $x_{\text {target }}$ was determined by processing the output signals from the azimuthal micro-compass mounted on the moving platform. The horizontal distance $x_{M A V}$ 


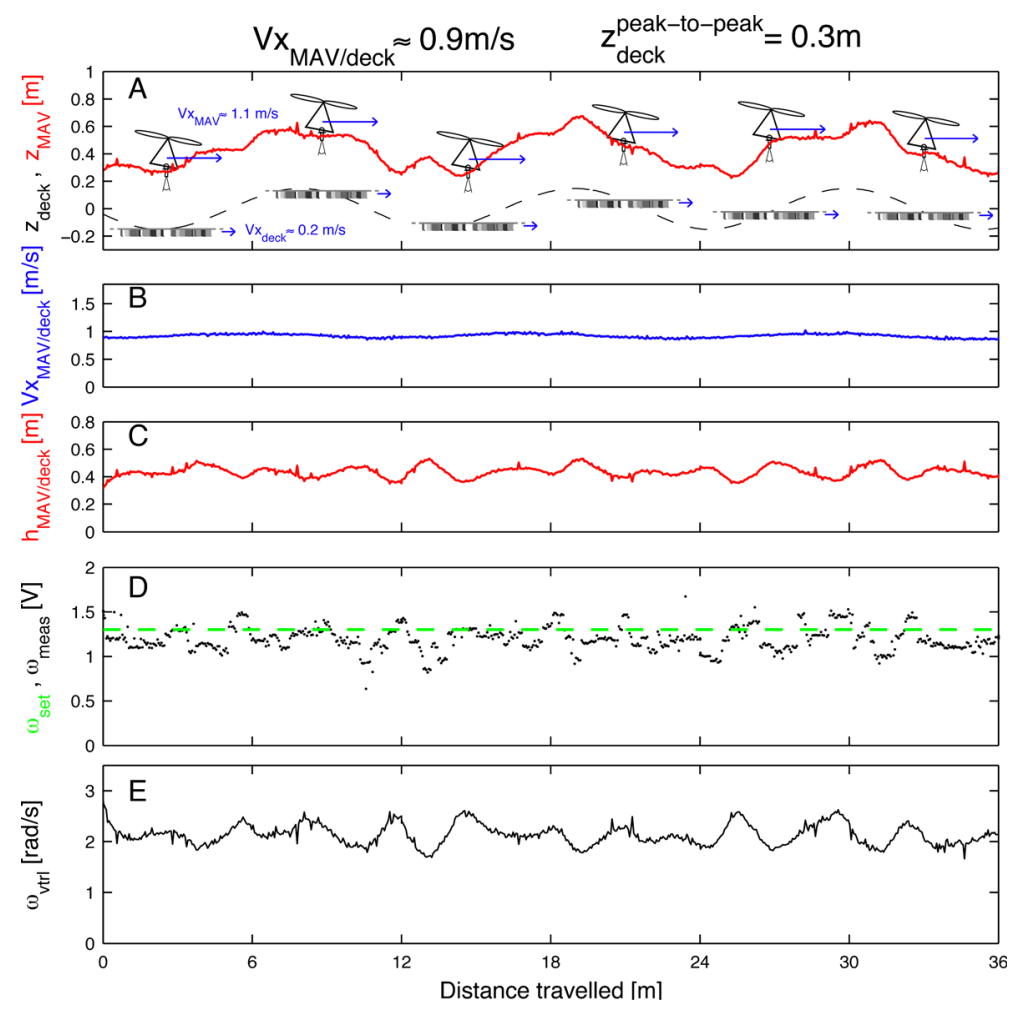

Fig. 6 Flight parameters of the robotic micro-helicopter flying over a platform that not only oscillated vertically but also moved forward at a constant speed. a Vertical trajectory $z_{M A V}$ of the MAV equipped with a ventral OF regulator (Fig. 3). The $0.3 \mathrm{~m}$-peak-to-peak amplitude vertical oscillation of the platform made the MAV automatically increase and decrease its altitude so as to keep the ventral OF constant. But the forward motion of the platform amounted to reducing the relative groundspeed of the MAV, causing the MAV to fly closer to the platform. b The groundspeed $V_{x_{M A V / d e c k}}$ is defined as the difference between the MAV's horizontal airspeed $V_{x_{M A V}}$ and the platform's horizontal speed $V_{x_{\text {deck }}}$. The robot's pitch was

actually travelled by the MAV was measured by processing the output signal from the optical encoder mounted on the azimuthal axis of the rotorcraft's supporting arm. The MAV's horizontal distance from the target $x_{M A V / \text { target }}$ was therefore computed as follows:

$x_{M A V / \text { target }}=x_{M A V}-x_{\text {target }}$

\subsection{Elevation Angle Feedback Loop}

To perform successful approach and landing maneuvers near the target, we added to the autopilot a second constant, giving an airspeed of $1.1 \mathrm{~m} / \mathrm{s}$. But since the deck itself moved forward $V_{x_{\text {deck }}}=+0.2 \mathrm{~m} / \mathrm{s}$, the MAV's relative groundspeed $V_{x_{M A V / d e c k}}$ was reduced to $0.9 \mathrm{~m} / \mathrm{s}$ throughout the journey. c The groundheight $h_{M A V / d e c k}$ was defined as the difference between the MAV's altitude $z_{M A V}$ and that of the platform, $z_{\text {deck }}$. Since the groundspeed was reduced here, the groundheight was observed to decrease as well and was only about $0.4 m$. It was maintained at about this value throughout the journey, in spite of the major dual perturbation (the vertical and horizontal movements of the platform) affecting the autopilot. d The output signals $\omega_{\text {meas }}$ delivered by the OF sensor again showed relatively small deviations from the OF set-point $\omega_{\text {set }}$

feedback loop (Fig. 9) focusing on the target's elevation angle. The main component of this second feedback loop was an elevation sensor (Fig. 8b) that was not physically realized but emulated for the sake of convenience (we are on the way to embedding a stand-alone target elevation sensor in the robot [47]). The reason for emulating the elevation angle sensor is that due to the circular shape of the flight arena, a classical camera could have seen the target and assessed its elevation angle only in the last two meters before landing which is a distance too short to properly test the second feedback control loop. With the virtual target's elevation sensor, the elevation angle feedback 

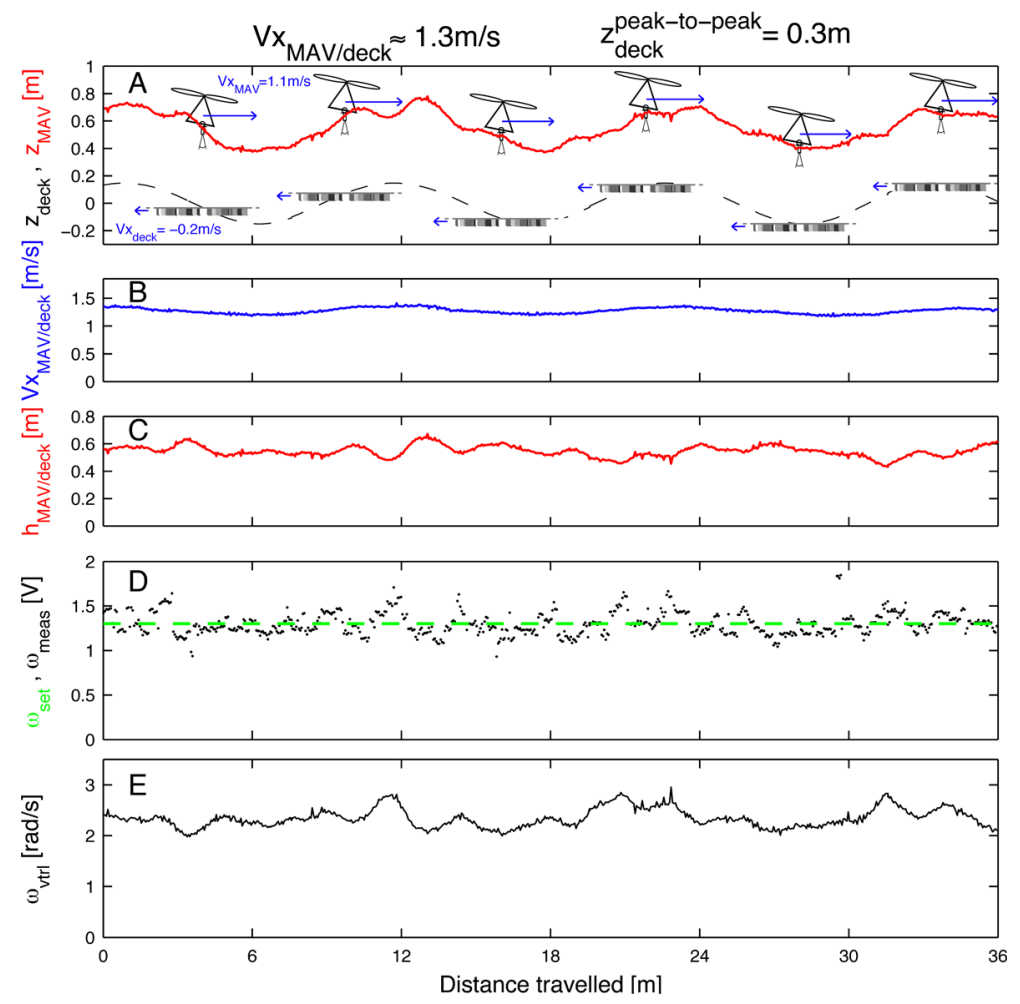

Fig. 7 Flight parameters of the robotic micro-helicopter flying over a platform that not only oscillated vertically but also moved backward at a constant speed. a Vertical trajectory $z_{M A V}$ of the MAV equipped with a ventral OF regulator (Fig. 3). The backward motion of the platform increased the relative groundspeed of the MAV (see b), causing the MAV to automatically fly further away (and consequently more safely) from the platform. b The robot's pitch was constant, giving a constant airspeed of $1.1 \mathrm{~m} / \mathrm{s}$. But since the platform moved backwards at the speed $V_{x_{\text {deck }}}=-0.2 \mathrm{~m} / \mathrm{s}$, the relative groundspeed $V_{x_{M A V / d e c k}}$

loop starts operating from a much longer distance. The virtual sensor calculated the target's elevation angle $\alpha$ on-line using the following equation:

$\alpha=\arctan \left(\frac{h_{M A V / \text { deck }}}{x_{M A V / \text { target }}}\right)$

According to equation 11, when the aerial robot is approaching the target, $x_{M A V / \text { target }}$ is reduced and then $\alpha$ could exceed the setpoint (see Fig. 10e) in case of small overshoot as $h_{M A V} /$ deck cannot be below $0.25 \mathrm{~m}$ because of the robot's landing gear.

This second feedback loop (Fig. 9) modified the MAV's pitch $\theta$ in the $0 \mathrm{deg}-5 \mathrm{deg}$ range so as to increased to $1.1-(-0.2)=+1.3 \mathrm{~m} / \mathrm{s}$ throughout the journey. c To keep the OF constant, the autopilot strived to adjust the lift so as to make the groundheight at all times proportional to the relative groundspeed. Since the groundspeed had increased, the groundheight increased as well and was always about $0.6 \mathrm{~m}$ throughout the journey, in spite of the vertical and horizontal movements of the platform. d The output signal $\omega_{\text {meas }}$ delivered by the OF sensor was monitored throughout the journey and again showed relatively small deviations from the OF set-point $\omega_{\text {set }}$. e So did the actual OF

decrease the MAV's airspeed when $\alpha$ increased. The elevation angle error signal $e_{\alpha}$ was delivered by a comparator to a proportional controller that modified the rotorcraft's pitch, and hence its horizontal airspeed, and hence its altitude (by virtue of the first feedback loop: the optic flow regulator), so as to bring the descent angle close to the set-point $\alpha_{\text {set }}$ while approaching the target. The operator did not have to intervene because the micro-helicopter decelerated automatically by decreasing its pitch $\theta$ (Fig. 8a) while approaching the target. In summary, the two intertwined feedback loops were such that:

- the first feedback loop (the OF regulator) modified the rotorcraft's lift, 


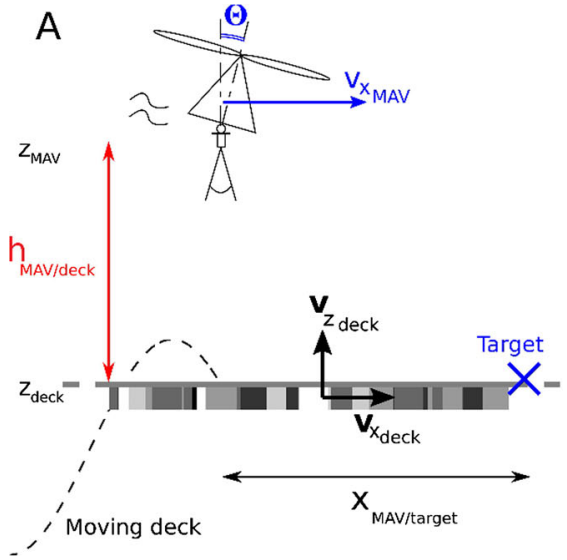

Fig. 8 Definition of the target's elevation angle $\alpha$ determined by a virtual sensor onboard the aerial robot flying in translation over a platform moving both horizontally and vertically. a In addition to the ventral OF, a virtual sensor onboard the aerial robot measured the elevation angle of the target. This angle $\alpha$ depended on the horizontal distance from the target $x_{M A V / \text { target }}$

- the second feedback loop (based on the elevation angle) modified the rotorcraft's horizontal speed.

These two feedback loops together made the microhelicopter land automatically near the target at a suitably low speed without any need for information about

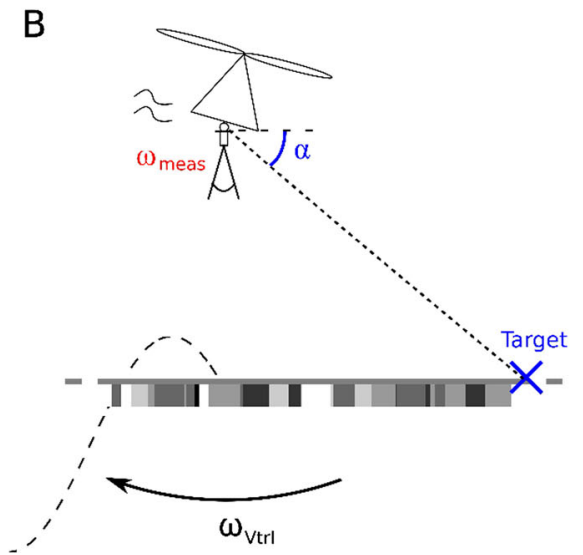

and the height $h_{M A V / d e c k}$ with respect to the surface below (see Eq. 11). b In addition to the output signal $\omega_{\text {meas }}$ delivered by the OF sensor mounted on the aerial robot, the autopilot used a virtual sensor able to determine the target elevation angle $\alpha$ : the latter was calculated on-line from the flight parameters given by the ground-truth sensors (see Eq. 11)

the altitude, speed, and distance travelled as shown in the video (Online Resource 1).

\subsection{Experimental Landing on the Moving Platform}

The target was placed on the moving platform at a virtual distance of about 40 meters from the aircraft.

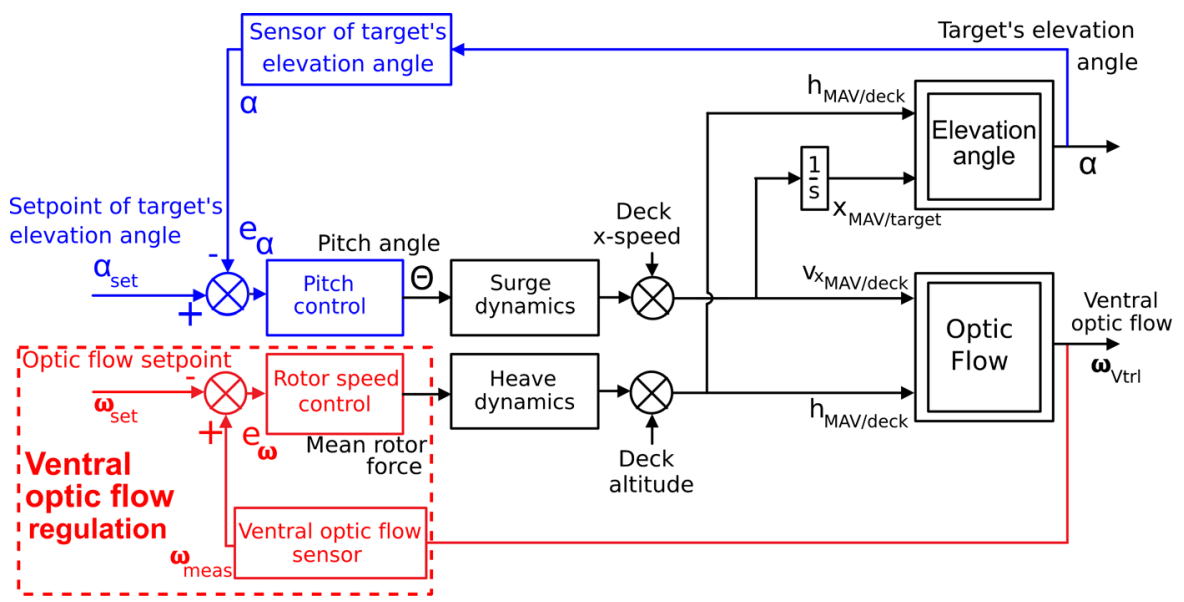

Fig. 9 Block diagram of the information flow showing the causal and dynamic relationships between the sensory and motor variables used to modify both the groundspeed and the groundheight so as to make the robot land safely near an oscillating target. In addition to the ventral $\mathrm{OF}$ regulator, which made the aerial robot adjust its lift, and hence its altitude at all times so as to keep the ventral OF $\omega_{\text {vtrl }}$ constant, a second feedback loop modified the rotorcraft's pitch $\theta$, consistently adjusting the horizontal speed as the robot approached the target. The diagram of the upper feedback loop shows how the target's elevation angle $(\alpha)$ was determined by a virtual sensor (the upper nonlinear box corresponds to $\alpha$, as described by (Eq. 11), and compared to an elevation angle set-point $\left(\alpha_{\text {set }}=10 \mathrm{deg}\right)$. The ensuing target error signal $\left(e_{\alpha}=\alpha-\alpha_{\text {set }}\right)$ drove a controller that would consistently adjust the rotorcraft's pitch $\theta$, so as to bring the descent angle close to the set-point while approaching the target 

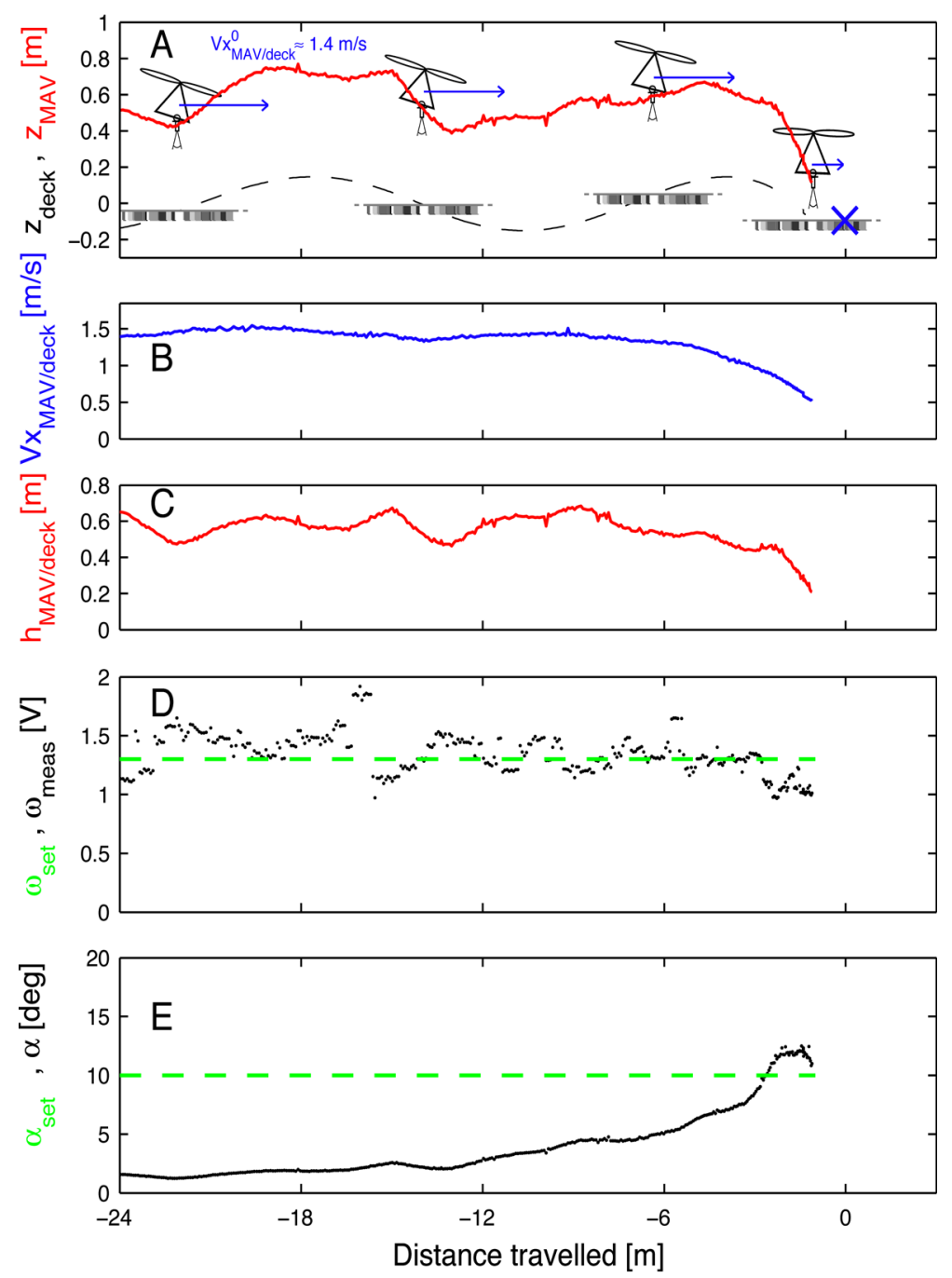

Fig. 10 Flight parameters monitored during the automatic landing of the micro-helicopter in the presence of vertical sinusoidal oscillations $\left(z_{\text {deck }}^{\text {peak-to-peak }}=0.3 \mathrm{~m}\right.$; frequency of $0.1 \mathrm{~Hz}$ ) of the platform on which the target was placed. The robot landed near the target after covering a distance of about 23 meters in about 20 seconds. It adjusted both its altitude and its speed to keep clear of the oscillating platform below, without ever crashing. The landing gear was $25 \mathrm{~cm}$ high. a Vertical trajectory $z_{M A V}$ of the MAV equipped with both a ventral OF regulator and a feedback loop based on the target's elevation angle (Fig. 9). The vertical oscillation of the platform makes the MAV automatically increase and decrease its altitude with the wave, so as to maintain the ventral OF constant. Upon approaching the target, the rotorcraft automatically

The target's elevation angle, which was therefore very small at the beginning of the experiment, gradually increased as the rotorcraft approached the target. Figures 10, 11 and 12 show the behavior of the rotor- decreases its pitch, and hence its speed relative to the platform, and hence its groundheight relative to the platform. b As the platform did not move horizontally here, the decrease in the groundspeed $V_{x_{M A V / d e c k}}$ which occurred upon approaching the target was only due to the increase in the target's elevation angle $\alpha$ (Fig. 9). c As the groundspeed decreased on approaching the target, the groundheight decreased proportionally. d The output signal $\omega_{\text {meas }}$ of the OF sensor showed a relatively small deviation from the OF set-point $\omega_{\text {set }}$ observed despite the large changes in the groundspeed, $V_{x_{M A V / d e c k}}$. e The virtual target elevation angle $\alpha$ calculated throughout the journey increased dramatically as the aircraft approached the target, until reaching the descent angle set-point (Fig. 9)

craft equipped with an OF regulator and a target elevation angle feedback loop controlling the altitude and the speed, respectively. The rotorcraft had to land near the target after flying over a non-stationary 

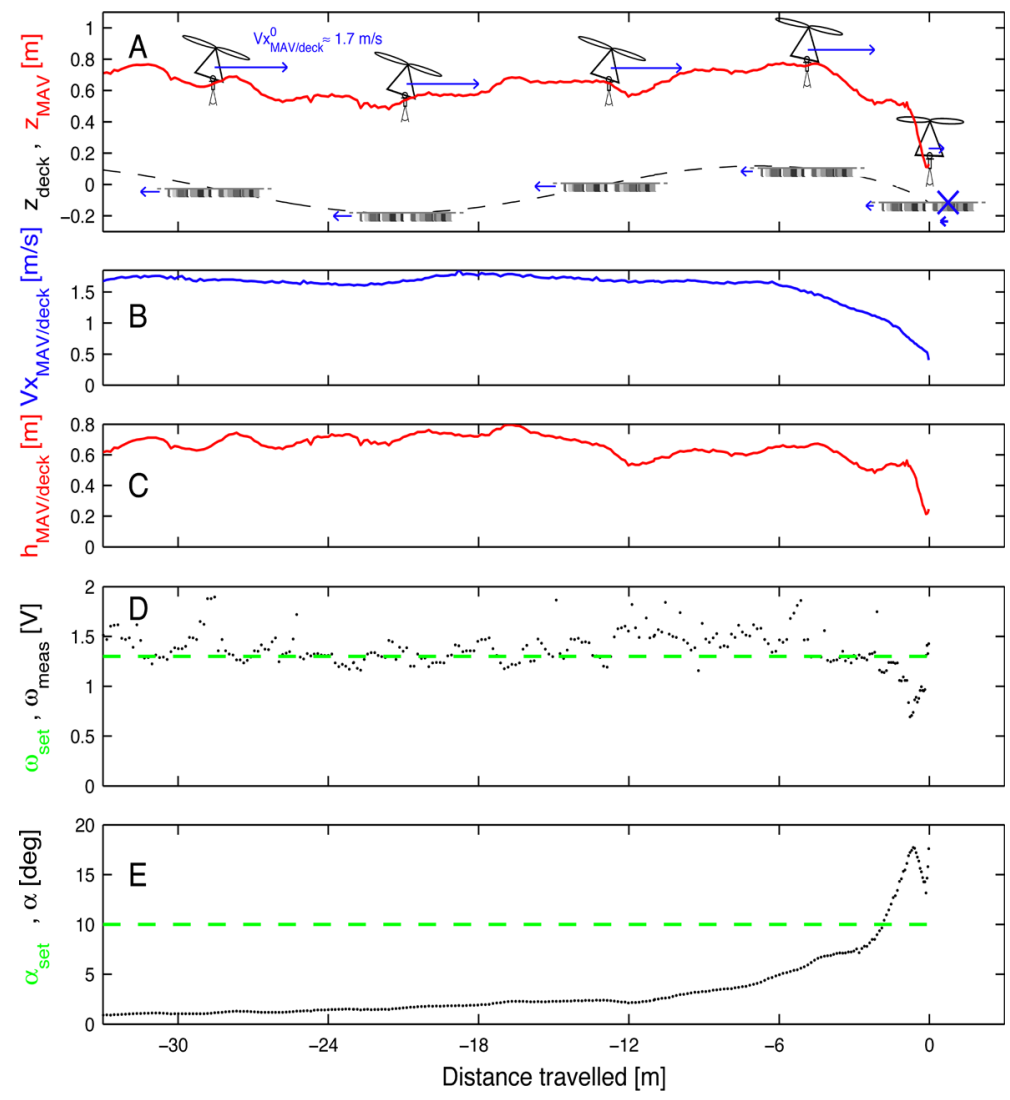

Fig. 11 Flight parameters monitored during the automatic landing of the micro-helicopter near the target, in the presence of two kinds of perturbations: a strong vertical sinusoidal oscillation of the platform $\left(z_{\text {deck }}^{\text {peak-to-peak }}=0.3 \mathrm{~m}\right.$; frequency: $0.05 \mathrm{~Hz}$ ) and a horizontal, backward motion of the platform at a declining speed (part of sinusoidal law with a peak to peak amplitude of $0.5 \mathrm{~m} / \mathrm{s}$ at a frequency of $0.01 \mathrm{~Hz}$ ). Despite these perturbations, the robot landed near the target after covering a distance of about 32 meters in about 30 seconds without ever crashing, by adjusting both its altitude and its speed to avoid the oscillating platform below. a Vertical trajectory $z_{M A V}$ of the MAV equipped with both a ventral OF regulator and a feedback loop based on the target's elevation angle (Fig. 9). Upon approaching the target, the rotorcraft automatically decreased

environment. During the approach and landing maneuvers, the platform moved up and down with a vertical amplitude of 0.3 meter and a vertical frequency of 0.05 or $0.1 \mathrm{~Hz}$, depending on the experiments. In all these experiments, the rotorcraft's airspeed $V_{x_{M A V}}$ resulted from its pitch angle $\theta$, which depended in turn on the target's elevation angle specified by the feedback loop. As the elevation angle increased upon approaching the target, the rotorcraft's its pitch, and hence its speed relative to the platform, and hence its altitude. b As the deck platform moved backward, the initially high groundspeed $V_{x_{M A V / d e c k}}$ gradually decreased upon approaching the target because of the gradual decrease of the platform's speed and the gradual increase of the target's elevation angle $\alpha$ (Fig. 9). c As the groundspeed decreased upon approaching the target, the groundheight decreased proportionally. d The output signal $\omega_{\text {meas }}$ of the OF sensor, which was monitored throughout the journey, showed a relatively small deviation from the OF set-point $\omega_{\text {set }}$ despite the large changes in the groundspeed $V_{x_{M A V / d e c k}}$. e The virtual target elevation angle $\alpha$ increased dramatically as the aircraft approached the target until the descent angle set-point was reached (Fig. 9)

second feedback loop decreased the pitch and hence the airspeed, and the OF regulator responded to this decrease in speed by decreasing the altitude proportionally until touchdown. In parallel, the ventral optic flow $\omega_{\text {vtrl }}$ generated by the vehicle was disturbed by the presence of the moving platform. The OF regulation loop rejected this perturbation by consistently adjusting the lift so as to restore the steady optic flow value (at the OF set-point). 

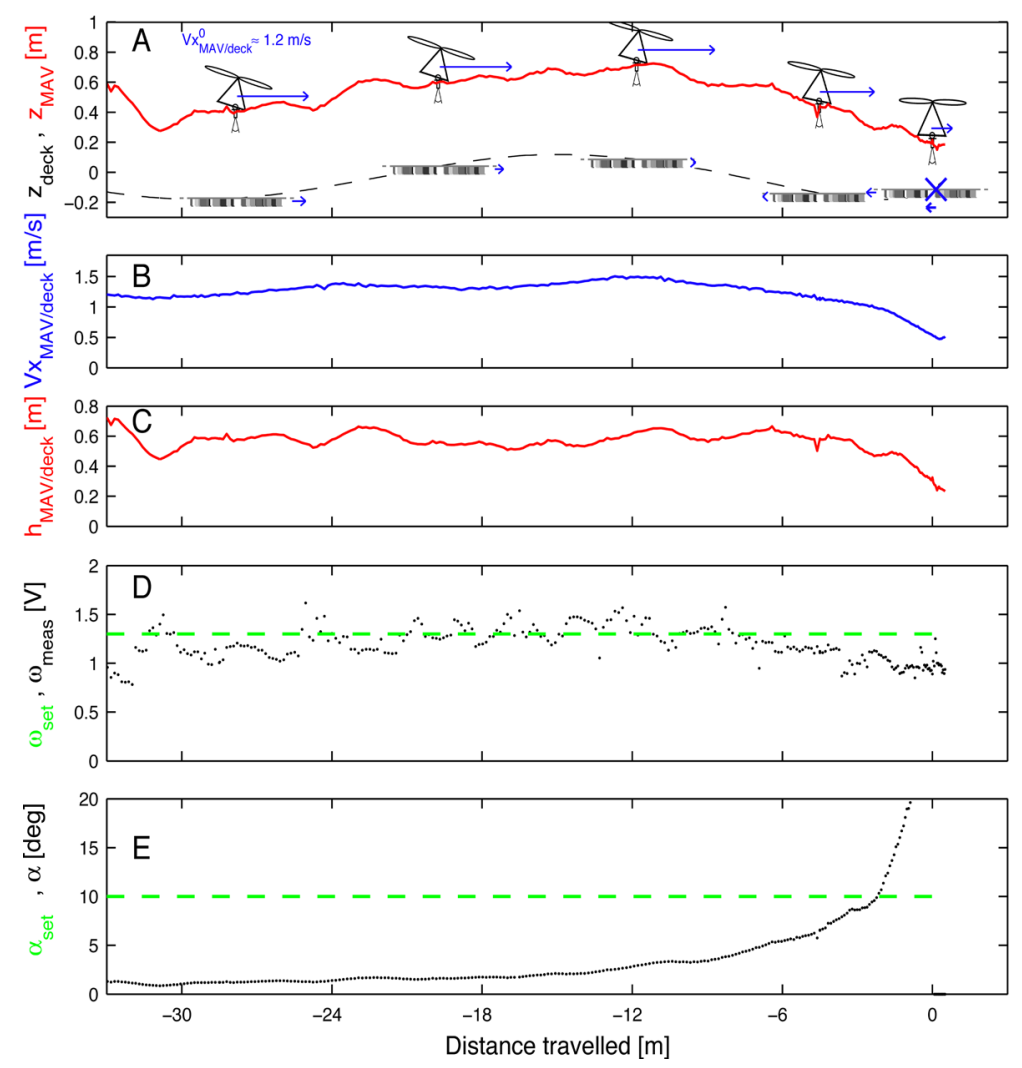

Fig. 12 Flight parameters monitored during the automatic landing of the micro-helicopter in the presence of strong vertical sinusoidal platform oscillations $\left(z_{\text {deck }}^{\text {peak-to-peak }}=0.3 \mathrm{~m}\right.$; frequency: $0.05 \mathrm{~Hz}$ ) as well as the sinusoidal forward and backward motion of the platform (amplitude: $0.5 \mathrm{~m} / \mathrm{s}$; frequency= $0.01 \mathrm{~Hz}$ ) on which the target was placed. The robot landed near the target after covering a distance of about 32 meters in about 30 seconds without ever crashing, by adjusting both its altitude and its speed to keep clear of the platform oscillating up and down as well as back and forth. a Vertical trajectory $z_{M A V}$ of the MAV equipped with a ventral OF regulator and a feedback loop based on the target's elevation angle. The vertical oscillation of the platform made the MAV automatically increase and decrease its altitude so as to keep the ventral OF constant. In parallel, upon approaching the target, the rotorcraft

In theory, the aerial robot cannot land precisely on the target by maintaining a 10-degree descent slope because of its landing gear. In the case the robot perfectly maintains its descent slope equal to the descent angle set-point $\left(\alpha_{\text {set }}=10 \mathrm{deg}\right)$ until touch down, the aerial robot will land at the distance before the target of $0.25 \mathrm{~m} / \tan (10 \mathrm{deg})$ corresponding to 1.42 automatically reduced its speed relative to the platform (via a reduction of its pitch), and hence its altitude. $\mathbf{b}$ The groundspeed then promptly decreased as the aircraft approached the target, due to the target's increasing elevation angle $\alpha$ (Fig. 9). c To keep the OF constant, the autopilot adjusted the lift to make the groundheight (relative to the platform) always proportional to the ground speed. As the groundspeed decreased upon approaching the target, the groundheight decreased proportionally. d The output signal $\omega_{\text {meas }}$ from the OF sensor showed a relatively small deviation from the OF set-point $\omega_{\text {set }}$ despite the occurrence of considerable changes in the groundspeed, $V_{x_{M A V / d e c k}}$. e The target elevation angle $\alpha$, which was monitored throughout the journey, increased dramatically as the aircraft approached the target, until reaching the descent angle set-point (Fig. 9)

meters. This is why this second feedback control law does not allow an absolute precise landing on the target but ensures a landing nearby the target using the elevation angle without a direct knowledge about the longitudinal distance to the target, as shown in Fig. 10 and in spite of additional disturbances as shown in Figs. 11 and 12. 
As the landing gear was devoid of tactile sensors the robot was not able to turn off its rotor immediately at touch down. The vibration occurring at touch down caused wrong optic flow measurements which sometimes made the robot rebound on the moving platform. We have therefore cut the landing trajectories at the time the robot touched the ground.

In Fig. 10, the platform was moving only vertically. The OF regulator can be seen to have nicely rejected the ventral OF perturbation by increasing or decreasing the rotorcraft's altitude $z_{M A V}$ as appropriate until touchdown occurred. As the rotorcraft approached the target, the groundheight relative to the platform decreased proportionally to the groundspeed relative to the platform in response to the signals received from the two intertwined feedback loops.

In Fig. 11, the platform was moving backward at a decreasing speed. The MAV's horizontal speed relative to the platform $\left(V_{x_{M A V / d e c k}}\right)$ was quite high up to a few meters from the target. As the platform was moving vertically as well, the aerial robot adjusted its altitude up and down and so as to keep the ventral OF constant. During the last few meters travelled before reaching the target, the rotorcraft promptly decreased its groundheight relative to the platform $h_{M A V / d e c k}$ in proportion to its speed relative to the platform $V_{x_{M A V / d e c k}}$.

In Fig. 12, the platform was set in alternating forward and backward motion. The MAV's horizontal speed relative to the platform $V_{x_{M A V / d e c k}}$ decreased as the rotorcraft began to approach the target. Since the platform was moving vertically as well in this case, the aerial robot consistently adjusted its altitude up and down so as to keep the ventral OF constant. In the final approach phase, the rotorcraft gradually decreased its groundheight relative to the platform $h_{M A V / \text { deck }}$ in proportion to the decrease in the speed relative to the platform $V_{x_{M A V / d e c k}}$.

All in all, the rotorcraft was able to fly safely and land on the platform that was moving sinusoidally both vertically and horizontally, by adjusting both its altitude (via the OF regulator) and its speed (via the target elevation angle feedback loop).

\section{Conclusion}

In the first part of this study, we established that the 'optic flow regulator" we had developed in former studies also allows a tethered rotorcraft to deal with non-stationary environments. This simple control system which strives to maintain the ventral OF constant made the aerial robot consistently follow a platform moving both vertically and horizontally as may occur, for instance, above the deck of a ship.

In the second part, we showed that the rotorcraft was able to land safely near a target placed on a platform that was moving both vertically and horizontally. The new autopilot presented here was based on two intertwined feedback loops:

- a ventral optic flow regulator driving the lift and hence the altitude,

- a second feedback loop based on the target's elevation angle, which drives the rotorcraft's pitch, and hence its forward thrust, and hence its forward airspeed.

The present study suggests that two relative cues (i) the ventral optic flow and (ii) the elevation angle relative to a target may suffice to enable a free-flying robot to navigate safely and even land in non-stationary environments. The performances of the aircraft landing on a moving platform equipped with the system presented here are reminiscent of those of birds and insects, which have long been performing complex visuomotor tasks in a completely autonomous manner. Sea-birds have no difficulty in landing on a moving ship or a floating tree-trunk moving with the seaswell. Honeybees have no difficulty either in landing on a dancing flower blown by the wind or an artificial moving target [18]. Flies are known to fixate their targets [48] and to land on them using visual cues [49]. Our biomimetic approach to robotic flight guidance in unsteady environments, which differs drastically from those based on more classical solutions, may help future flying robots to acquire some autonomy at a very low cost: the present tethered robot did not need any inputs about the groundspeed, groundheight, platform speed or platform height, for instance, to be able to land safely on a vertically and horizontally oscillating target. The present system works with one proviso, however. Because an OF sensor is a non-emissive sensor relying on photons and contrast, it may fail at night or under conditions giving poor visibility (in fog, dust, rain, or snow), where it would not be able to compete with active sensors such as FLIR, Laser or Millimeter Wave Radars. But photons and contrast are available in many terrestrial (and planetary) environments. 
Should the contrast deteriorate in the visible range for whatever reason (such as dust storms), it might still be available in other parts of the electromagnetic spectrum (in the UV to Tera-Hertz range), so that it might still be possible to measure the OF. In any case, the simple sensory and electronic control systems adopted in this study provides evidence that the bio-inspired solution of using optic flow cues can be scaled up to an aerial robot whose Reynolds number is way beyond that of flying insects. Once further engineered, these systems promise dramatic savings in terms of autonomy, payload, onboard sensor suites, onboard processing, and onboard energy resources. Systems of this kind may even prove to be highly suitable for use on micro-air vehicles (MAVs) down to insect-scale robots [11], as well as on micro-space vehicles (MSVs), while providing backup or alert systems on conventional aircraft and spacecraft. We now plan to apply this bio-inspired strategy to free-flying aircraft as well as to planetary landing situations in collaboration with the European Space Agency (ESA) [50].

Acknowledgment The authors are grateful to the three reviewers for their helpful suggestions about the manuscript and thank S. Viollet, J. Serres, G. Desilles and G. Portelli for their helpful comments and suggestions during this work, M. Boyron for his outstanding technical assistance with the electronics, D. Dray, F. Paganucci and Y. Luparini for their outstanding assistance with the mechanics and micromechanics, F. Roubieu, J. Diperi, R. Juston and F. Expert for their assistance in taking the photographs of the experimental set-up, S. Amic for the design of the EMD circuit, M. Rigal for helping with the galvanic isolation of the dSpace board, and J. Blanc for her assistance with the English manuscript. This Work was supported partly by CNRS Institutes (Life Science; Information Science; Engineering Science and Technology), by Aix-Marseille University, by an EU contract (IST/FET -1999- 29043) and by the French Defense Agency (DGA, 0534 022).

Open Access This article is distributed under the terms of the Creative Commons Attribution License which permits any use, distribution, and reproduction in any medium, provided the original author(s) and the source are credited.

\section{References}

1. Ruffier, F., Franceschini, N.: Optic flow regulation: the key to aircraft automatic guidance. Robot. Auton. Syst. 50(4), 177-194 (2005)

2. Franceschini, N., Ruffier, F., Serres, J.: A bio-inspired flying robot sheds light on insect piloting abilities. Curr. Biol. 17, 329-335 (2007)
3. Sousa, P., Wellons, L., Colby, G., Walters, J., Weir, J.: Test results of an f/a-18 automatic carrier landing using shipboard relative global positioning system. Technical report, Naval Air Warfare Center Aircraft Division, Tech. Rep (2003)

4. Reboulet, C.: Appontage automatique d'un uav -onera desd toulouse-. In: Proceedings of the JNRR'99, Montpellier (1999)

5. Coutard, L., Chaumette, F., Pflimlin, J.M.: Automatic landing on aircraft carrier by visual servoing. In: IEEE/RSJ Int. Conf. on Intelligent Robots and Systems, IROS'11. San Francisco, pp. 2843-2848 (2011)

6. Vu, B., Lemoing, T., Costes, P.: Integration of flight and carrier landing aid systems for shipboard operations. In: AGARD, Aircraft Ship Operations 15 (1991)

7. Subrahmanyam, M.: H infinity design of f/a-18a automatic carrier landing system. Journal of Guidance. Control Dyn. 17(1), 187-191 (1994)

8. Zufferey, J.C., Floreano, D.: Fly-inspired visual steering of ultralight indoor aircraft. IEEE Trans. Robot. 22(1), 137146 (2006)

9. de Croon, G., Ho, H., Wagter, C.D., van Kampen, E., Remes, B., Chu, Q.: Optic-flow based slope estimation for autonomous landing. In: International Micro Air Vehicle Conference and Flight Competition (IMAV2013). Toulouse (2013)

10. Briod, A., Zufferey, J.C., Floreano, D.: Optic-flow based control of a $46 \mathrm{~g}$ quadrotor. In: Workshop on Vision-based Closed-Loop Control and Navigation of Micro Helicopters in GPS-denied Environments. IROS, Tokyo (2013)

11. Ma, K., Chirarattananon, P., Fuller, S., Wood, R.: Controlled flight of a biologically inspired, insect-scale robot. Science 340(6132), 603-607 (2013)

12. Voskuijl, M., Padfield, G., Walker, D., Manimala, B., Gubbels, A.: Simulation of automatic helicopter deck landings using nature inspired flight control and flight envelope protection. Aeronaut. J. 114(1151): Paper No. 3426 (2008)

13. Saripalli, S., Montgomery, J., Sukhatme, G.: Visually guided landing of an unmanned aerial vehicle. IEEE Trans. Robot. Autom. 19(3), 371-380 (2003)

14. Saripalli, S., Sukhatme, G.S.: Landing a helicopter on a moving target. In: Proceedings of 2007 IEEE International Conference on Robotics and Automation (2007)

15. Edwards, B., Archibald, J., Fife, W., Lee, D.J.: A vision system for precision MAV targeted landing. In: International Symposium on Computational Intelligence in Robotics and Automation (CIRA 2007), 20-23 June 2007, pp. 125-130 (2007)

16. Rui, W., Guangjun, Z., Peng, Y.: Optical flow based 3d motion estimation for autonomous landing an uav on deck. In: Proceedings of SPIE, the International Society for Optical Engineering ISSN 0277-786X CODEN PSISD, International conference on space information technology (19-20 November, 2005, Wuhan, China), vol. 5985 (2006)

17. Portelli, G., Ruffier, F., Franceschini, N.: Honeybees change their height to restore their optic flow. J. Comp. Physiol. A. 196(4), 307-313 (2010)

18. Zhang, S., Wang, X., Liu, Z., Srinivasan, M.: Visual tracking of moving targets by freely flying honeybees. Vis. Neurosci. 4(4), 379-86 (1990) 
19. Iida, F.: Goal-directed navigation of an autonomous flying robot using biogically inspired cheap vision. In: Proceedings of the 32nd International Symposium on Robotics, pp. 1404-1409. ISR, Seoul (2001)

20. Green, W., Oh, P., Barrows, G.: Flying insect inspired vision for autonomous aerial robot maneuvers in near-earth environments. In: Proceeding of IEEE International Conference of Robotics and Automation (ICRA), pp. 2347-2352. New Orleans (2004)

21. Beyeler, A., Zufferey, J.C., Floreano, D.: Vision-based control of near-obstacle flight. Auton. Robot. 27(3), 201-219 (2009)

22. Zufferey, J.C., Beyeler, A., Floreano, D.: Autonomous flight at low altitude using light sensors and little computational power. Int. J. Micro Air Veh. 2(2), 107-117 (2010)

23. Netter, T., Franceschini, N.: A robotic aircraft that follows terrain using a neuromorphic eye. In: Proceedings of IEEE Conference on Intelligent Robots and Systems (IROS), Lausanne (2002)

24. Ruffier, F., Viollet, S., Amic, S., Franceschini, N.: Bioinspired optical flow circuits for the visual guidance of micro-air vehicles. In: Proceedings of IEEE Int. Symposium on Circuits and Systems (ISCAS), Vol. III, pp. 846-849. Bangkok (2003)

25. Ruffier, F., Franceschini, N.: Aerial robot piloted in steep relief by optic flow sensors. In: International Conference on Intelligent Robots and Systems (IROS), pp. 1266-1273. Nice (2008)

26. Herisse, B., Russotto, F., Hamel, T., Mahony, R.: Hovering flight and vertical landing control of a VTOL unmanned aerial vehicle using optical flow. In: Proceedings of IEEE/RSJ International Conference on Intelligent Robots and Systems (IROS), pp. 1404-1409. Nice (2008)

27. Kendoul, F., Nonami, K., Fantoni, I., Lozano, R.: An adaptive vision-based autopilot for mini flying machines guidance, navigation and control. Auton. Robot. 27(3), 165-188 (2009)

28. Viollet, S., Franceschini, N.: Biologically-inspired visual scanning sensor for stabilization and tracking. In: Proceeding IEEE/RSJ International Conference on Intelligent Robots and System (IROS), pp. 204-209. Kyongju (1999)

29. Kerhuel, L., Viollet, S., Franceschini, N.: Steering by gazing: An efficient biomimetic control strategy for visually guided micro aerial vehicles. IEEE Trans. Robot. 26, 307319 (2010)

30. Garratt, M., Chahl, J.: Vision-based terrain following for an unmanned rotorcraft. J. Field Robot. 25, 284-301 (2008)

31. Garcia-Carrillo, L.R., Flores, G., Sanahuja, G., Lozano, R.: Quad-rotor switching control: An application for the task of path following. In: American Control Conference (ACC). Montreal (2012)

32. Conroy, J., Gremillion, G., Ranganathan, B., Humbert, J.: Implementation of wide-field integration of optic flow for autonomous quadrotor navigation. Auton. Robot. 27(3), 189-198 (2009)
33. Hérissé, B., Hamel, T., Mahony, R., Russotto, F.X.: A terrain-following control approach for a vtol unmanned aerial vehicle using average optical flow. Auton. Robot. 29(3-4), 381-399 (2010)

34. Griffiths, S., Saunders, J., Curtis, A., Barber, B., McLain, T., Beard, R.: Maximizing miniature aerial vehicles. IEEE Robot. Autom. Mag. 13, 34-43 (2006)

35. Mellinger, D., Michael, N., Kumar, V.: Trajectory generation and control for precise aggressive maneuvers with quadrotors. Int. J. Robot. Res. 31(5), 664-674 (2012)

36. Shen, S., Mulgaonkar, Y., Michael, N., Kumar, V.: Visionbased state estimation for autonomous rotorcraft mavs in complex environments. In: IEEE International Conference on Robotics and Automation (ICRA). Karlsruhe, pp. 17581764 (2013)

37. Herisse, B., Hamel, T., Mahony, R., Russotto, F.X.: The landing problem of a vtol unmanned aerial vehicle on a moving platform using optical flow. In: Proceedings of the 2010 IEEE/RSJ International Conference on Intelligent Robots and Systems. Taipei, p. 2010

38. Herisse, B., Hamel, T., Mahony, R., Russotto, F.X.: Landing a vtol unmanned aerial vehicle on a moving platform using optical flow. IEEE Trans. Robot. 28(1), 77-89 (2012)

39. Wenzel, K.E., Masselli, A., Zell, A.: Automatic take off, tracking and landing of a miniature uav on a moving carrier vehicle. J. Intell. Robot. Syst 61, 221-238 (2011)

40. Webb, B.: Can robots make good models of biological behavior? Behav. Brain Sci. 24, 1033-1050 (2001)

41. Kennedy, J.S.: The migration of the desert locust (schistocerca gregaria forsk.) Phil. Trans. Royal Soc. B 235, 163$290(1951)$

42. Koenderink, J., van Doorn, A.: Facts on optic flow. Biol. Cybern. 56, 247-254 (1987)

43. Pichon, J.M., Blanes, C., Franceschini, N.: Visual guidance of a mobile robot equipped with a network of self-motion sensors. In: Wolfe, W.J., Chun, W.H. (eds.) Proceedings of SPIE Conf. on Mobile Robots IV, Vol. 1195, pp. 44-53. SPIE, Bellingham (1989)

44. Ruffier, F., Franceschini, N.: Octave, a bioinspired visuomotor control system for the guidance of micro-air vehicles. In: Rodriguez-Vazquez, A., Abbott, D., Carmona, R. (eds.) SPIE Vol. 5119, Bioengineered and Bioinspired Systems, pp. 1-12. SPIE, Bellingham (2003)

45. Ruffier, F., Franceschini, N.: Visually guided micro-aerial vehicle : automatic take off, terrain following, landing and wind reaction. In: Proceeding of IEEE International Conference on Robotics and Automation (ICRA). New Orleans, pp. 2339-2346 (2004)

46. Ruffier, F.: Pilote Automatique Biomimétique, Système générique inspiré du contrôle visuomoteur des insectes pour : le décollage, le suivi de terrain, la réaction au vent et 1 ' atterrissage automatiques d' un micro-aéronef. $\mathrm{PhD}$ thesis, INP Grenoble, Ecole Doctorale EEATS, Spécialité : Signal, Image, Parole, Télécommunications (2004)

47. Ruffier, F., Benacchio, S., Expert, E., Ogam, E.: A tiny directional sound sensor inspired by crickets designed for 
micro-air vehicles. In: Proceeding of IEEE Sensors 2011 conference, Limerick, Ireland, pp. 970-973 (2011)

48. Reichardt, W., Wenking, H.: Optical detection and fixation of objects by fixed flying flies (Musca domestica). Naturwissenschaften 56, 424-425 (1969)

49. van Breugel, F., Dickinson, M.H.: The visual control of landing and obstacle avoidance in the fruit fly, drosophila melanogaster. J. Exp. Biol. 215, 1783-1798 (2012)

50. Valette, F., Ruffier, F., Viollet, S., Seidl, T.: Biomimetic optic flow sensing applied to a lunar landing scenario. In: Proceedings of IEEE International Conference on Robotics and Automation (ICRA 2010), pp. 2253-2260 (2010) 